\title{
Inhibition of Notch1 protects against IL-1 $\beta$-induced inflammation and cartilage destruction in temporomandibular chondrocytes
}

\author{
WANGGUI YING ${ }^{1,2}$, FANG YUAN ${ }^{1,2}$, PING $\mathrm{HE}^{2}$ and PING JI ${ }^{1}$ \\ ${ }^{1}$ Shandong Provincial Key Laboratory of Oral Tissue Regeneration, School of Stomatology, \\ Shandong University; ${ }^{2}$ Jinan Stomatological Hospital, Jinan, Shandong 250012, P.R. China
}

Received March 4, 2016; Accepted February 13, 2017

DOI: $10.3892 / \mathrm{mmr} .2017 .6511$

\begin{abstract}
Temporomandibular disorder (TMD) is a complex and multifactorial disease, with inflammatory response and cartilage destruction usually associated with its etiology. Notch1 is a type I transmembrane receptor, which is critical in determining the growth, differentiation and survival of various cell types. However, its role and mechanism in interleukin (IL)-1 $\beta$-stimulated temporomandibular chondrocytes remain to be elucidated. In the present study, chondrocytes were isolated from Sprague-Dawley rats and stimulated by IL-1 $\beta$ at a concentration of $10 \mathrm{ng} / \mathrm{ml}$, and the expression of Notch1 was inhibited by small interfering RNA. The expression levels of Notch1 and Notch1 intracellular domain (NICD) were analyzed using reverse transcription-quantitative polymerase chain reaction and western blot analyses. Matrix metalloproteinases (MMPs) and the tissue inhibitor of metalloproteinases (TIMPs) were assessed using western blot analysis, and nuclear factor- $\kappa \mathrm{B}(\mathrm{NF}-\kappa \mathrm{B})$ was also analyzed. Compared with the control, IL-1 $\beta$ stimulation increased the expression of Notch1 and NICD. In addition, IL-1 $\beta$ stimulation increased the secretion of tumor necrosis factor- $\alpha$ and IL-6, and the expression of intercellular adhesion molecule 1 and inducible nitric oxide synthase. By contrast, inhibition of Notch1 reduced these inflammatory responses by suppressing the nuclear translocation and phosphorylation of NF- $\mathrm{KB}$ p65. The inhibition of Notch1 also reduced the expression of MMPs and increased the expression of TIMP-1. These data suggested that the inhibition of Notch1 prevented the IL-1 $\beta$-induced inflammatory response partly by inhibiting the NF-KB signaling pathway in temporomandibular chondrocytes. The inhibition of Notch1
\end{abstract}

Correspondence to: Dr Ping Ji, Shandong Provincial Key Laboratory of Oral Tissue Regeneration, School of Stomatology, Shandong University, 44-1 Wenhuaxi Road, Jinan, Shandong 250012, P.R. China

E-mail: jiping@sdu.edu.cn

Key words: Notch1 signaling, temporomandibular chondrocytes, interleukin-1 $\beta$, inflammation response, cartilage destruction suppressed cartilage destruction by modulating the balance between MMPs and TIMP-1, therefore, inhibition of Notch1 may be a mechanism for the treatment of TMD.

\section{Introduction}

Temporomandibular disorder (TMD) comprises a series of conditions, which cause pain and dysfunction in the muscles and joints between the lower jaw and the base of the skull. TMD is a complex and multifactorial disease, with physical, social and psychological factors usually associated with its etiology $(1,2)$. Although progress has been made, the precise pathogenesis of TMD remains to be fully elucidated, which leads to difficulty in diagnosis and therapy. Usually, the temporomandibular cartilage withstands pressures of frequent jaw movement and biting hard food (3). Abnormal elevated intra-articular pressure and local inflammatory response are important in TMD. The differential expression of inflammatory cytokines has been found in patients with TMD, including monocyte chemotactic protein 1, interleukin (IL)-1 and IL-8 (4). Inflammation can also stimulate the production of matrix metalloproteinases (MMPs), downregulate the expression of tissue inhibitor of metalloproteinases (TIMPs) and accelerate the degradation of cartilage matrix (5). An imbalance between MMPs and TIMPs has been reported to be important in the progression of TMD (6).

IL-1 is a potent pro-inflammatory cytokine, which functions as the gatekeeper for inflammation (7). It is produced by a variety of cells and acts on almost every organ system of the body. IL-1 consists of two distinct proteins (IL-1 $\alpha$ and IL-1 $\beta$ ), which bind to the type 1 IL-1 receptor to activate a downstream pro-inflammatory pathway (8). IL-1 $\beta$, a cytokine released by synovial cells and macrophages, causes inflammation of the articular cartilage (9).

The Notch signaling pathway consists of a family of four type I transmembrane receptors (Notch1-4), which undergo proteolytic processing by a furin-like convertase during transit to the cell surface (10). It is important in the growth, differentiation and survival of various cell types in diverse tissues (11). In the canonical signaling pathway, binding of a ligand triggers sequential receptor cleavage, and results in the release and translocation of the Notch1 intracellular domain (NICD) 
to the nucleus, where it functions as a transcriptional activator $(12,13)$. Several lines of evidence have demonstrated that Notch is important in regulating the responsiveness of immune cells to stimulation and infection $(14,15)$. Furthermore, inflammatory mediators can increase the expression of Notch (16), and the upregulation of Notch signaling elevates responses to interferon- $\gamma$, leading to increased expression of inflammatory cytokines in macrophages $(17,18)$. However, the role of Notch1 signaling in IL-1 $\beta$-stimulated temporomandibular chondrocytes remains to be elucidated.

The present study investigated whether the inhibition of Notch1 is able to inhibit inflammatory responses and cartilage destruction in TMD by establishing an in vitro model using temporomandibular chondrocytes.

\section{Materials and methods}

Isolation and culture of chondrocytes. The temporomandibular chondrocytes were isolated from the articular cartilage of 15 male Sprague-Dawley rats (6-week-old; 140-160 g). Animal care and all procedures were performed according to institutional guidelines and were approved by the Ethics Committee of Shandong University (Shangdong, China). All animals were housed in the same room on a $12 \mathrm{~h}$ light:dark cycle at $22^{\circ} \mathrm{C}$ and had full access to standard chow and water. All surgical procedures were performed under sodium pentobarbital anesthesia and all efforts were made to minimize suffering. Briefly, the harvested cartilage was minced and incubated in a trypsin-containing solution for $2 \mathrm{~h}$ at $37^{\circ} \mathrm{C}$. The minced cartilage was then washed with phosphate-buffered saline (PBS; Sigma-Aldrich; KGaA Millipore, Darmstadt, Germany) and incubated with $0.2 \%$ collagenase (Sigma-Aldrich; KGaA Millipore) at $37^{\circ} \mathrm{C}$ overnight. Following digestion, the chondrocytes were collected via centrifugation at $800 \mathrm{x}$ g for $15 \mathrm{~min}$ at $37^{\circ} \mathrm{C}$ and cultured in Dulbecco's modified Eagle's medium (DMEM; Gibco; Thermo Fisher Scientific, Inc., Waltham, MA, USA) containing 10\% fetal calf serum (Gibco; Thermo Fisher Scientific, Inc.), $100 \mathrm{IU} / \mathrm{ml}$ penicillin and $100 \mu \mathrm{g} / \mathrm{ml}$ streptomycin (Gibco; Thermo Fisher Scientific, Inc.). The cells were serum-starved overnight prior to treatment. Cells in passages 1-3 were used in all experiments.

Cell treatment and gene inhibition. The primary temporomandibular chondrocytes were cultured at a density of 5,000 cells/well in DMEM at $37^{\circ} \mathrm{C}$ in an atmosphere of $5 \% \mathrm{CO}_{2}$ and treated with $10 \mathrm{ng} / \mathrm{ml} \mathrm{IL-1} \beta$ (Sigma-Aldrich; KGaA Millipore). The cells were harvested following incubation for $24 \mathrm{~h}$. To inhibit the expression of Notch1, the cells were transfected with Notch1 small interfering (s)iRNA (GenePharma, Shanghai, China) or negative control siRNA using Lipofectamine 2000 (Invitrogen; Thermo Fisher Scientific, Inc.). Experiments were performed $24 \mathrm{~h}$ following transfection. The cells in the Notch1 group were stimulated with IL-1 $\beta$ following inhibition of Notch1 by siRNA.

Nuclear protein extraction. The nuclear proteins of the chondrocytes were extracted using an NE-PER Nuclear and Cytoplasmic Extraction Reagent kit (Pierce; Thermo Fisher Scientific, Inc.) according to the manufacturer's protocol. The protein was quantified using the bicinchoninic acid (BCA) method (Bio-Rad Laboratories, Inc., Hercules, CA, USA). All extracts were stored at $-80^{\circ} \mathrm{C}$ until use.

Enzyme-linked immunosorbent assay (ELISA). The inflammatory cytokines in the cell culture supernatant were measured using ELISA kits for tumor necrosis factor (TNF)- $\alpha$ and IL-6 (R\&D Systems, Inc., Minneapolis, MN, USA), as described previously (19). All spectrophotometric readings were performed using an absorption spectrometer (Thermo Fisher Scientific, Inc.).

Measurement of $m R N A$ expression using reverse transcription-quantitative polymerase chain reaction ( $R T-q P C R)$ analysis. Total RNA was extracted from the cultured cells using TRIzol (Invitrogen; Thermo Fisher Scientific, Inc.) and reverse transcribed to cDNA using SYBR-Green Supermix (Bio-Rad Laboratories, Hercules, CA, USA). The $1 \mu \mathrm{g}$ mRNA levels were measured using qPCR analysis. The thermocycling conditions for PCR amplification were as follows: $95^{\circ} \mathrm{C}$ for $5 \mathrm{~min}, 36$ cycles at $95^{\circ} \mathrm{C}$ for $10 \mathrm{sec}$, annealing at $56^{\circ} \mathrm{C}$ for $30 \mathrm{sec}$ and elongation at $72^{\circ} \mathrm{C}$ for $30 \mathrm{sec}$. The primer sequences were as follows: Notch1, forward 5'-CGGGTCCACCAGTTT GAATG-3' and reverse 5'-GTTGTATTGGTTCGGCAC CAT-3'; intercellular adhesion molecule 1 (ICAM-1), forward 5'-TTGGAAGCC-TCATCCG-3' and reverse 5'-CAATGTTG CGAGACCC-3'; inducible nitric oxide synthase (iNOS), forward 5'-GTTCTCAGCCCAACAATACAAGA-3' and reverse 5'-GTGGACGGGTCGATGTCAC-3'; GAPDH, forward 5'-GGATGACCTTGCCCACAGCCT-3' and reverse 5'-ATCTCTGC-CCCCTCTGCTGA-3'. qPCR was performed on the iCycler real-time PCR system (Bio-Rad Laboratories, Inc.) and relative quantification was performed using the comparative quantification $(\mathrm{Cq})$ method (20).

Western blot analysis. The concentrations of protein extracted from chondrocytes were quantified using the BCA method with a protein assay kit (Beyotime Institute of Biotechnology, Nantong, China). The samples $(50 \mu \mathrm{g})$ were separated on $10 \%$ SDS-PAGE and electrophoretically transferred onto a polyvinylidene difluoride membrane (GE Healthcare Life Sciences, Piscataway, NJ, USA). The blots were blocked for $2 \mathrm{~h}$ with $5 \%$ non-fat dry milk in Tris-buffered saline at room temperature and then incubated overnight with anti-Notch1 (catalog no. 36085; 1:1,000), anti-NICD (catalog no. 4147; 1:1,000), anti-ICAM (catalog no. 4915; 1:1,000), anti-iNOS (catalog no. 13120; 1:1,000) obtained from Cell Signaling Technology, Inc., Danvers, MA, USA. Additional antibodies used were anti-MMP-1 (catalog no. ab137332; 1:500, Abcam, Cambridge, MA, USA), anti-MMP-9 (catalog no. 2270; 1:2,000), anti-TIMP-1 (catalog no. 8946; 1:1,000), anti-nuclear factor (NF)-кB p65 (catalog no. 8242; 1:1,000), anti-phosphorylated (p)-NF- $\kappa$ B p65 (catalog no. 4806; 1:1,000), anti-histone (catalog no. 7631; 1:1,000) and anti- $\beta$-actin antibodies (catalog no. 3700; 1:1,000) all obtained from Cell Signaling Technology, Inc., at $4^{\circ} \mathrm{C}$. Following washing with $0.1 \%$ TBS-Tween 20, the membranes were incubated with secondary horseradish peroxidase-conjugated IgG (catalog nos. ZB-2301 and ZB-2305; 1:1,000; ZSGB-BIO, Beijing, China) at room temperature for $2 \mathrm{~h}$. The blots were then visualized using 
A

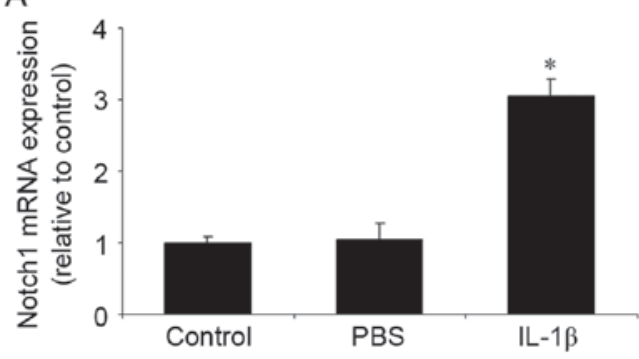

C

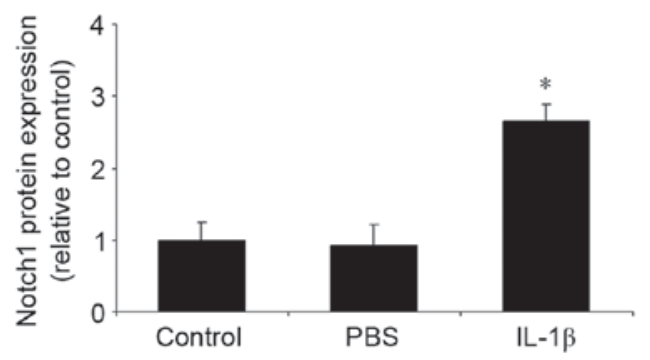

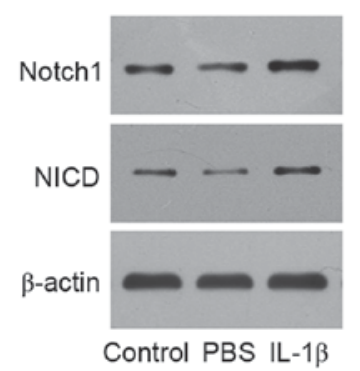

D

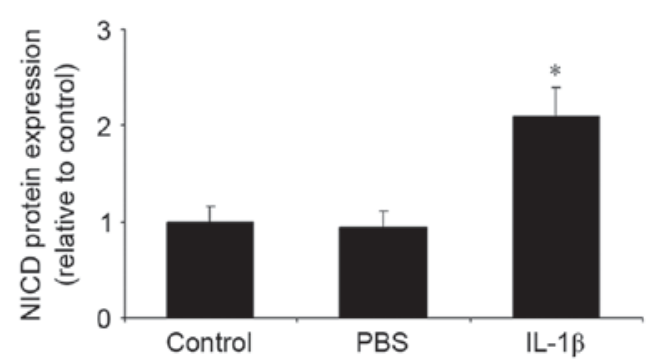

Figure 1. IL-1 $\beta$ increases the expression of Notch1 and NICD. Following stimulation of temporomandibular chondrocytes with IL-1 $\beta$, the expression of Notch1 and NICD were assessed using reverse transcription-quantitative polymerase chain reaction and western blot analyses IL-1 $\beta$ stimulation increased the (A) mRNA and (B and C) protein expression levels of Notch1. (D) IL-1 $\beta$ stimulation increased the protein expression of NICD. Values are expressed as the mean \pm standard deviation from three independent experiments. "P<0.05, vs. control. IL-1 $\beta$, interleukin- $1 \beta$; NICD, Notch1 intracellular domain; PBS, phosphate-buffered saline.

A

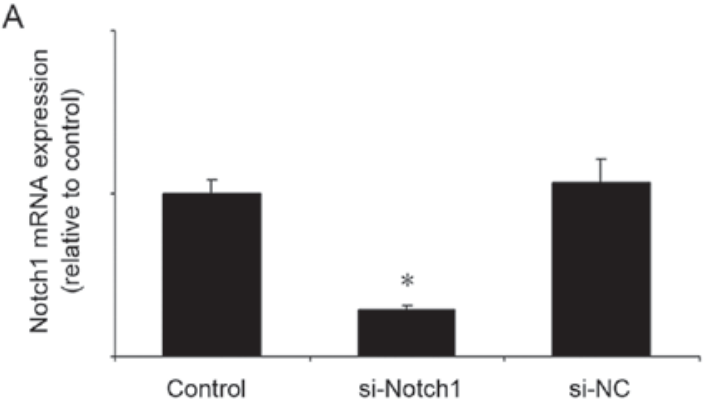

C

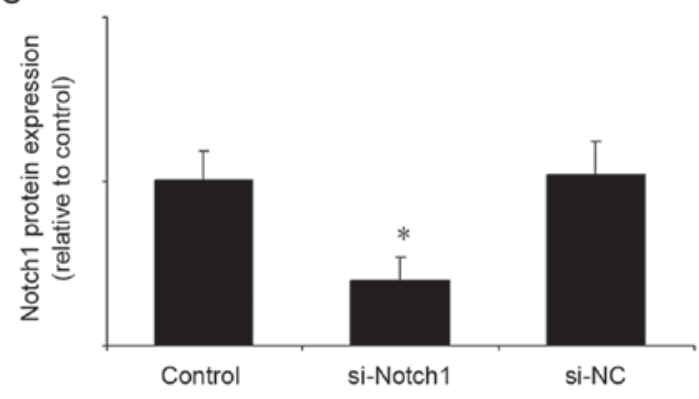

B Notch1

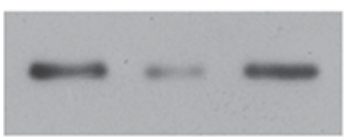

$\beta$-actin

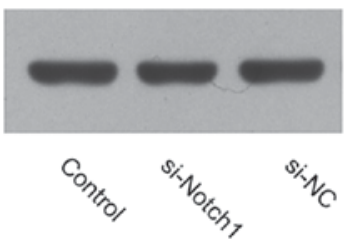

Figure 2. Notch1 siRNA reduces the mRNA and protein expression of Notch1. Following inhibition of Notch1 by siRNA, the mRNA and protein expression levels of Notch1 were assessed using reverse transcription-quantitative polymerase chain reaction and western blotting analyses. (A) Notch1 siRNA reduced the mRNA expression of Notch1. (B and C) Notch1 siRNA reduced the protein expression of Notch1, whereas si-NC had no effect. Values are expressed as the mean \pm standard deviation. ${ }^{*} \mathrm{P}<0.05$, vs. control. si, small interfering RNA; NC, negative control.

enhanced chemiluminescence with ECL reagents (Pierce; Thermo Fisher Scientific, Inc.).

Statistical analysis. Data are presented as the mean \pm standard deviation from three independent experiments. SPSS software, version 13.0 (SPSS, Inc., Chicago, IL, USA) was used for statistical analysis. Intergroup comparisons were performed using Student's t-test (two-tailed) or one-way analysis of variance. $\mathrm{P}<0.05$ was considered to indicate a statistically significant difference.

\section{Results}

$I L-1 \beta$ increases the expression of Notchl and NICD. Following stimulation of the temporomandibular chondrocytes with IL-1 $\beta$, the expression levels of Notch1 and NICD were measured using RT-qPCR and western blot analyses. As shown in Fig. 1A-D, no differences were found between the control group and the PBS group. However, compared with the control group, IL-1 $\beta$ significantly increased the mRNA and protein expression levels of Notch1, and the protein expression of NICD. 
A

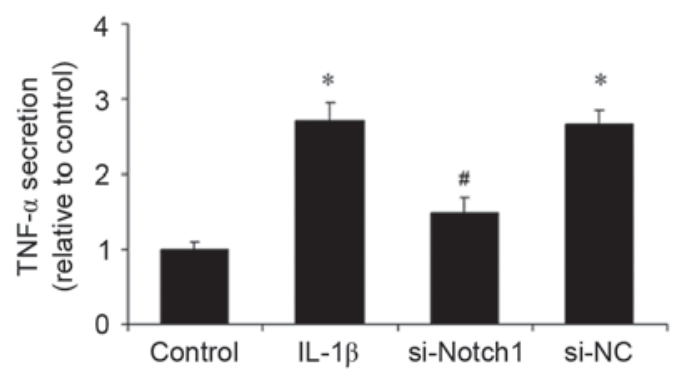

B

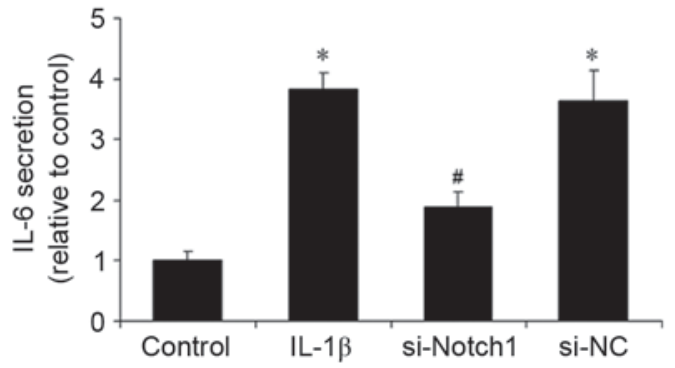

Figure 3. Inhibition of Notch1 reduces IL-1 $\beta$-induced secretion of TNF- $\alpha$ and IL- 6 . The temporomandibular chondrocytes were stimulated with IL-1 $\beta$ following inhibition of Notch1 using siRNA. The secretion of TNF- $\alpha$ and IL- 6 was assessed using an enzyme-linked immunosorbent assay. (A) Inhibition of Notch1 reduced IL-1 $\beta$-induced secretion of TNF- $\alpha$. (B) Inhibition of Notch1 reduced IL-1 $\beta$-induced secretion of IL-6. Values are expressed as the mean \pm standard deviation. ${ }^{*} \mathrm{P}<0.05$, vs. control; ${ }^{\#} \mathrm{P}<0.05$, vs. IL-1 $\beta$. TNF- $\alpha$, tumor necrosis factor- $\alpha$; IL, interleukin; si, small interfering RNA; NC, negative control.
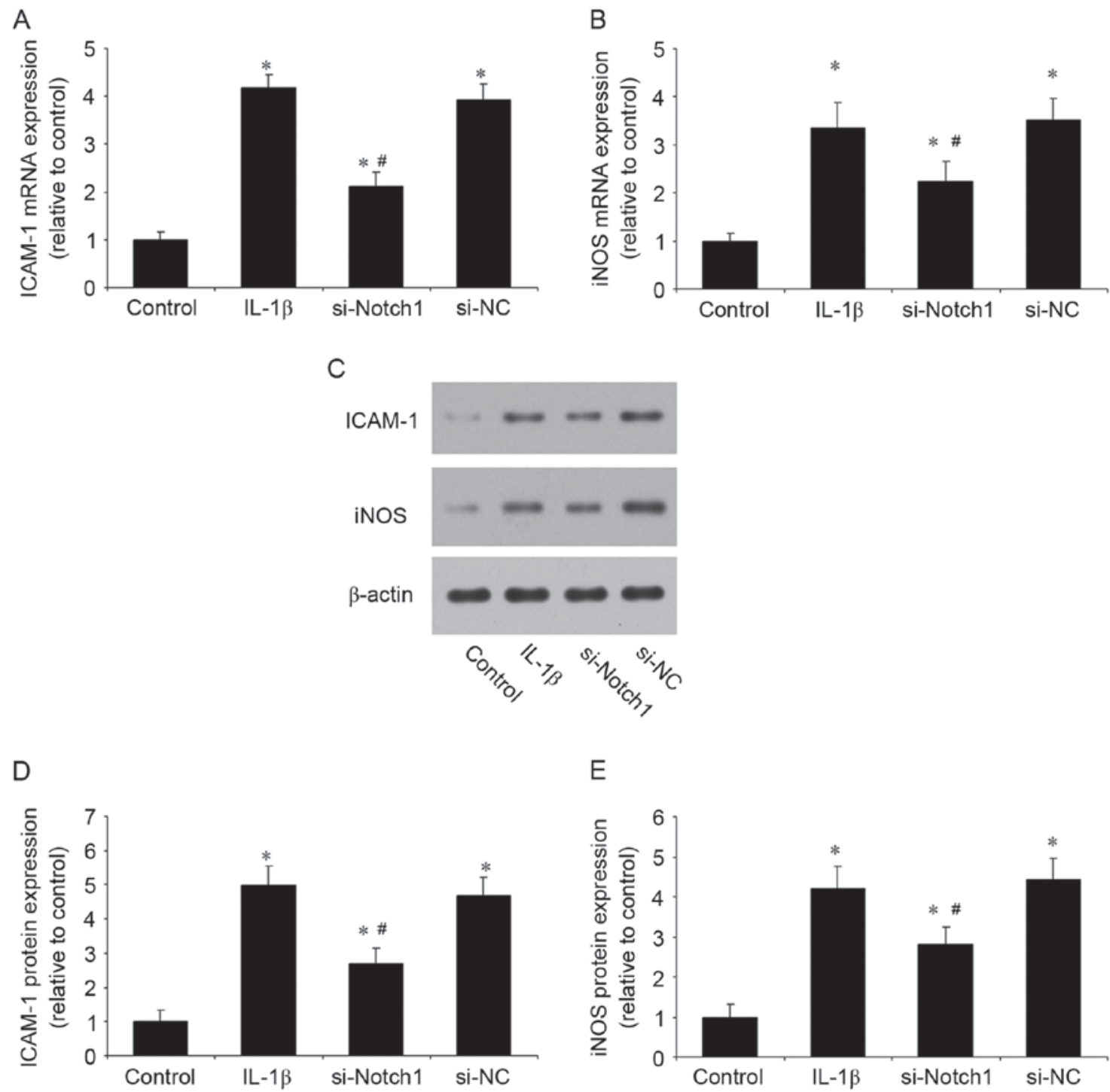

Figure 4. Inhibition of Notch1 reduces IL-1 $\beta$-induced inflammatory cytokine expression. The temporomandibular chondrocytes were stimulated with IL-1 $\beta$ following inhibition of Notch1 using siRNA, The expression levels of ICAM-1 and iNOS were assessed using reverse transcription-quantitative polymerase chain reaction and western blot analyses. Inhibition of Notch1 reduced the IL-1 $\beta$-induced mRNA expression of (A) ICAM-1 and (B) iNOS. (C) Inhibition of Notch1 reduced the IL-1 $\beta$-induced protein expression of (D) ICAM-1 and (E) iNOS. Values are expressed as the mean \pm standard deviation. *P $<0.05$, vs. control; ${ }^{\#} \mathrm{P}<0.05$, vs. IL-1 $\beta$. si, small interfering RNA; NC, negative control; ICAM-1, intercellular adhesion molecule 1; iNOS, inducible nitric oxide synthase.

Notchl siRNA reduces the $m R N A$ and protein expression of Notch1. In the subsequent experiments, the expression of
Notch1 was inhibited by siRNA. The efficiency of Notch1 siRNA was first verified. Compared with the control group, 
A

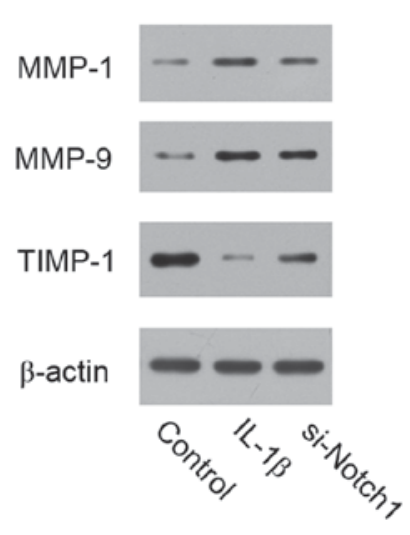

C

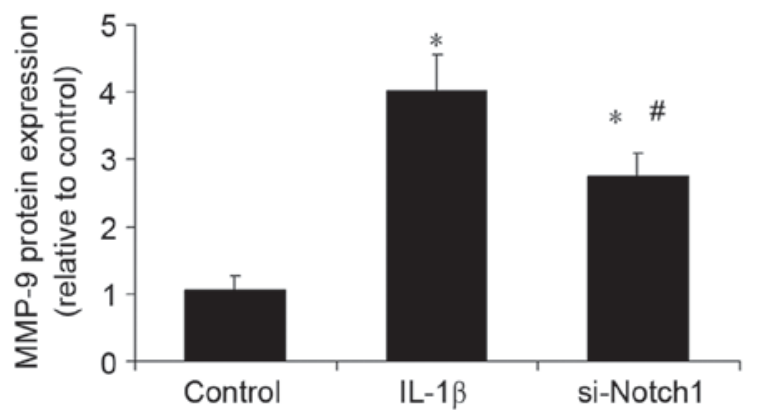

B

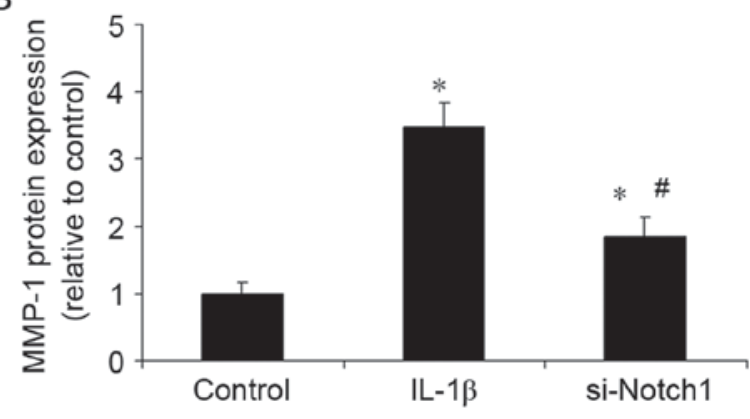

D

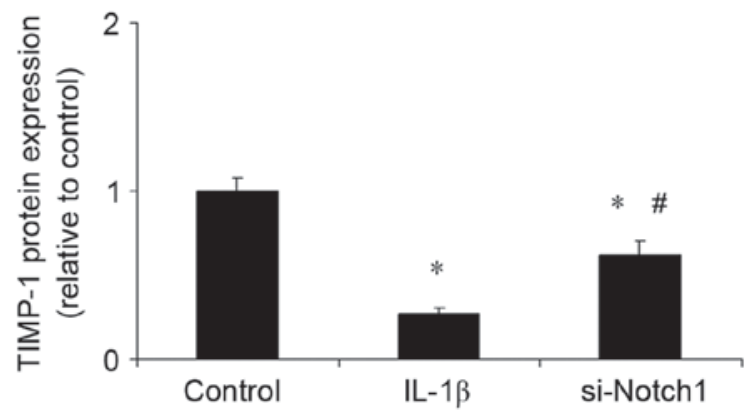

Figure 5. Inhibition of Notch1 reduced MMPs and increases the protein expression of TIMP-1. The temporomandibular chondrocytes were stimulated with IL-1 $\beta$ following inhibition of Notch1 by siRNA. (A) Protein expression levels of MMPs and TIMP-1 were determined using western blot analysis. Compared with the control, IL-1 $\beta$ stimulation increased the expression of (B) MMP-1 and (C) MMP-9, and reduced the expression of (D) TIMP-1. However, inhibition of Notch1 reduced the expression of MMPs and increased the expression of TIMP-1. Values are expressed as the mean \pm standard deviation. $\mathrm{P}<0.05$, vs. control; ${ }^{\#} \mathrm{P}<0.05$, vs. IL-1ß. MMP, matrix metalloproteinase; TIMP-1, tissue inhibitor of metalloproteinase-1; si, small interfering RNA; NC, negative control.

Notch1 siRNA significantly reduced the mRNA (Fig. 2A) and protein (Fig. 2B and C) expression levels of Notch1, whereas the negative control siRNA had no effect.

Notchl inhibition reduces the $I L-1 \beta$-induced inflammatory response. Inflammation is important in the pathogenesis of TMD. In the present study, following stimulation of the chondrocytes with IL-1 $\beta$, the secretion of TNF- $\alpha$ and IL-6 were examined, as was the mRNA and protein expression levels of ICAM-1 and iNOS. Stimulation with IL-1 $\beta$ increased the levels of TNF- $\alpha$ and IL- 6 in the supernatants, whereas the inhibition of Notch1 by siRNA reduced these levels and control siRNA had no effect (Fig. 3A and B). In addition, the inhibition of Notch1 significantly reduced the IL-1 $\beta$-induced mRNA (Fig. 4A and B) and protein (Fig. 4C-E) expression levels of ICAM-1 and iNOS (Fig. 4). These results suggested that the inhibition of Notch1 suppressed the IL-1 $\beta$-induced inflammatory response in chondrocytes.

Notchl inhibition reduces the expression of MMPs and increases the expression of TIMP-1. An imbalance between MMPs and TIMPs has been confirmed to be important in the progression of TMD. The present study investigated the effect of inhibiting Notch1 on the expression levels of MMPs and TIMP-1. Compared with the control, IL-1 $\beta$ stimulation increased the expression levels of MMP-1 and MMP-9 (Fig. 5A-C), and reduced the expression of TIMP-1 (Fig. 5D). However, the inhibition of Notch1 had the opposite effect, reducing the expression levels of MMPs and increasing the expression of TIMP-1.

Notchl inhibition suppresses IL-1 $\beta$ induced activation of $N F-\kappa B$ in chondrocytes. The present study also investigated whether the protective effects of inhibiting Notch1 involved the $\mathrm{NF}-\mathrm{kB}$ signaling pathway. The nuclear translocation of NF- $\kappa \mathrm{B}$ p65 was analyzed using western blot analysis. As shown in Fig. 6A-D, the localization of NF- $\kappa \mathrm{B}$ p65 was predominantly cytoplasmic prior to IL-1 $\beta$ stimulation, however, it was translocated to the nucleus when cells were stimulated with IL-1 $\beta$. The inhibition of Notch1 suppressed this IL-1 $\beta$-induced p65 nuclear translocation. The expression of p-p65 was also measured. Compared with the control, IL-1 $\beta$ increased the expression of p-p65 3-fold, whereas inhibition of Notch1 reduced the IL-1 $\beta$-induced expression of p-p65 (Fig. 6E and F). These results suggested that the protective effect of Notch1 inhibition occurred, at least in part, through the NF-KB signaling pathway.

\section{Discussion}

TMD is an assorted set of clinical conditions characterized by pain and dysfunction of the masticatory muscles in the temporomandibular joint (TMJ) (21). The prevalence of TMD varies between 5 and $86 \%$ depending on different epidemiological studies, diagnostic criteria and methods of assessment (22). According to the latest understanding, inflammatory cytokines are the most important pathogenic 
A

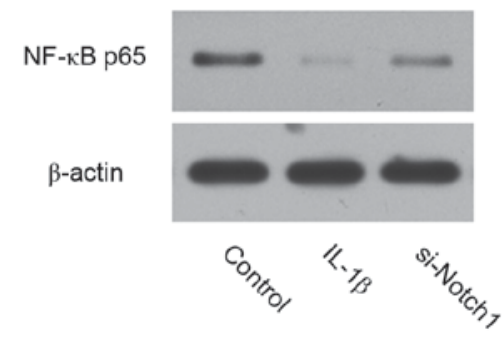

C

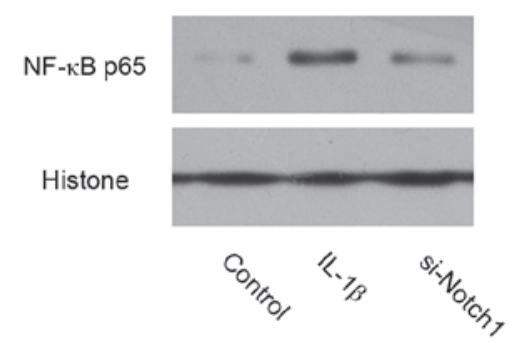

E

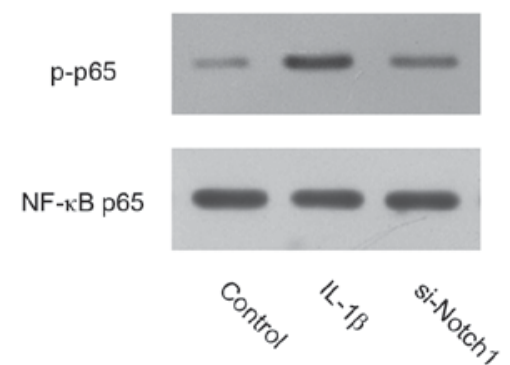

B

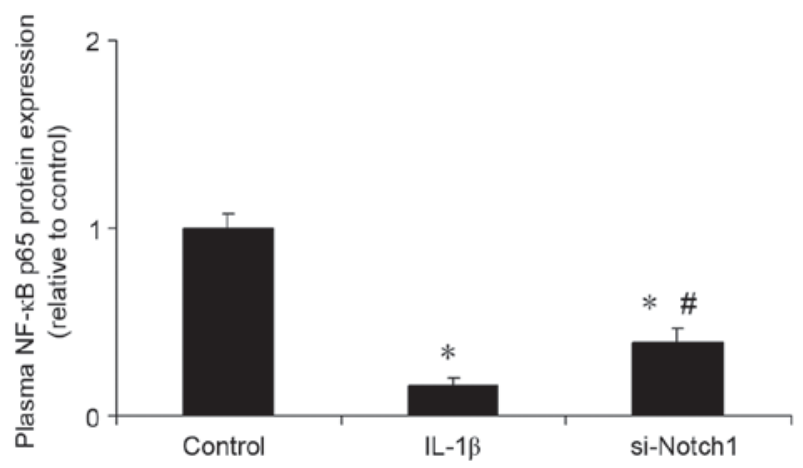

$\mathrm{D}$

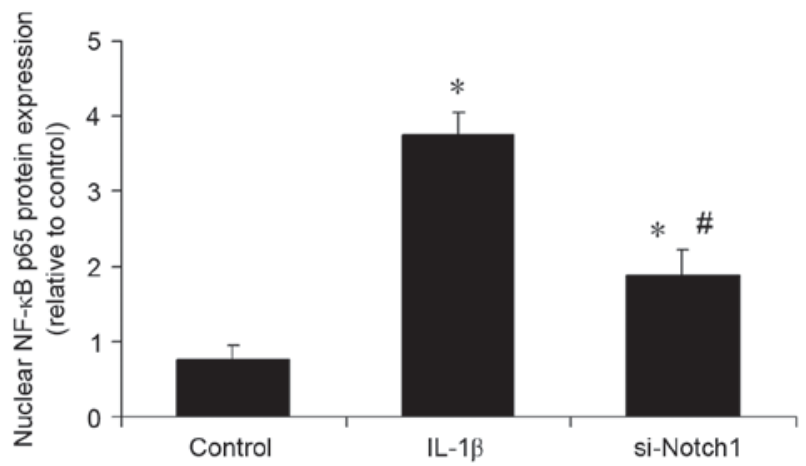

$\mathrm{F}$

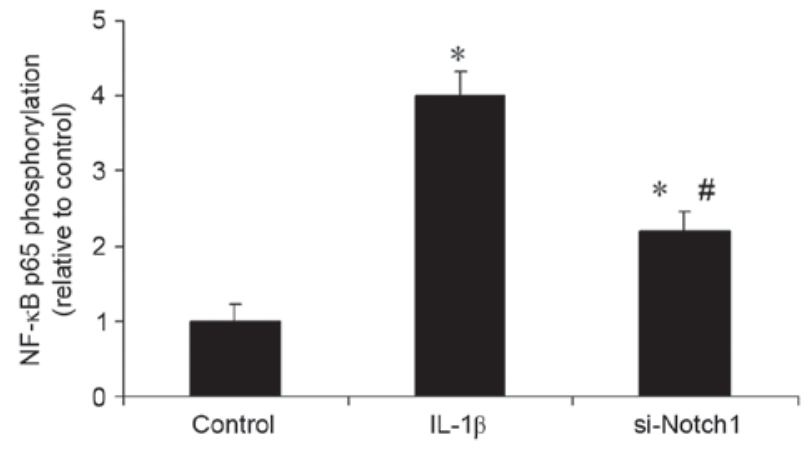

Figure 6. Inhibition of Notch1 suppresses the IL-1 $\beta$-induced nuclear translocation and phosphorylation of NF- $\kappa$ B p65. Following inhibition of Notch1 with siRNA, the cells were stimulated by IL-1 $\beta$, and the expression and phosphorylation of NF- $\mathrm{B}$ p 65 were analyzed using western blot analysis. Inhibition of Notch1 reduced the IL-1 $\beta$-induced nuclear translocation of $\mathrm{NF}-\kappa \mathrm{B}$ p65, resulting in (A and B) an increased expression in plasma and (C and D) decreased expression in the nucleus. (E and F) Inhibition of Notch1 reduced the IL-1 $\beta$-induced phosphorylation of NF- $\mathrm{B}$ p65. Values are expressed as the mean \pm standard deviation. ${ }^{*} \mathrm{P}<0.05$, vs. control; ${ }^{\#} \mathrm{P}<0.05$, vs. IL-1 $\beta$. NF- $\kappa \mathrm{B}$, nuclear factor- $\kappa \mathrm{B}$; IL-1 $\beta$, interleukin-1 $\beta$; si, small interfering RNA; NC, negative control.

factors involved in the pathogenesis of TMD (23), responsible for the loss of metabolic homeostasis by promoting catabolic and destructive processes. Among the numerous representatives of these inflammatory cytokines, the highest importance is attributed to IL-1 $\beta$, TNF- $\alpha$ and IL-6. In the present study, temporomandibular chondrocytes were stimulated with IL-1 $\beta$. Compared with the control group, IL-1 $\beta$ significantly induced the inflammatory response in vitro, including the secretion of TNF- $\alpha$ and IL-6, and mRNA and protein expression of ICAM-1 and iNOS. These results were accordance with previous findings in vivo (24).

MMPs are a family of $>20$ metalloenzymes, which are crucial in tissue remodeling and cartilage degradation due to their ability to degrade extracellular matrix (25). TIMPs are considered to be specific inhibitors of MMPs. An imbalance between the activity of MMPs and their inhibitors is considered to underlie cartilage destruction. The expression of MMP-2 and MMP-9 has been reported to increase in the rat trigeminal ganglion during the development of TMJ inflammation (26). In the present study, it was found that IL-1 $\beta$ stimulation increased the expression of MMP-1 and MMP-9, and reduced the expression of TIMP-1, whereas the inhibition of Notch1 by siRNA reduced the expression of MMPs and increased the expression of TIMP-1. Therefore, maintaining the balance of MMPs/TIMP-1 is the precise mechanism by which the inhibition of Notch1 prevents cartilage matrix degradation. This prompted investigation of the probable cell signaling transduction. 
$\mathrm{NF}-\kappa \mathrm{B}$ is pivotal in regulating inflammation-associated genes (27). Various stimuli, including pro-inflammatory cytokines, lead to the nuclear translocation of NF- $\mathrm{B}$ p65 and subsequently to the upregulated transcription of various proinflammatory cytokines, including IL-1, TNF $\alpha$, IL-6 and IL-8 (28). NF- $\kappa$ B remains inactive in the cytoplasm through interaction with the inhibitory protein, inhibitor $\kappa \mathrm{B}(\mathrm{I} \kappa \mathrm{B})$. The activation of $N F-\kappa B$ requires the phosphorylation and subsequent degradation of $\mathrm{I} \kappa \mathrm{B} \alpha$ (29). It has been demonstrated that the phosphorylation of p65 is critical for binding to its target sites on DNA (30). Considering $N F-\kappa B$ is a pivotal regulator of the inflammatory response, the cross-talk between IL-1 $\beta$ and $\mathrm{NF}-\kappa \mathrm{B}$ in chondrocytes may be one of the major causes of TMJ inflammation. As expected, stimulation with IL-1 $\beta$ in the present study induced the nuclear translocation and phosphorylation of NF- $\mathrm{B}$ p 65, with an increased expression of inflammatory cytokines, including TNF $\alpha$, IL-6, ICMA-1 and iNOS.

In conclusion, the results of the present study provide novel insights into the regulatory mechanisms of Notch1 in temporomandibular chondrocytes during TMD. IL-1 $\beta$ stimulation induced Notch1, and subsequent variation was associated with $\mathrm{NF}-\kappa \mathrm{B}$ pathway, which may be an important pathogenetic factor in the development of TMD. The present study, to the best of our knowledge, is the first to provide evidence that the inhibition of Notch1 effective treated TMD. However, further in vivo experiments are required to identify novel and potent agents for the prevention and treatment of TMD through the inhibition of Notch1.

\section{Acknowledgements}

This study was supported by the Science and Technology Development Plans of Shandong Province (grant no. 2014GSF118027) and the Specialized Research Fund for the Doctoral Program of Higher Education of China (grant no. 20120131110074).

\section{References}

1. Reissmann DR, John MT, Schierz O and Wassell RW: Functional and psychosocial impact related to specific temporomandibular disorder diagnoses. J Dent 35: 643-650, 2007.

2. Kiener HP and Karonitsch T: The synovium as a privileged site in rheumatoid arthritis: Cadherin-11 as a dominant player in synovial pathology. Best Pract Res Clin Rheumatol 25: 767-777, 2011

3. Wu M, Xu T, Zhou Y, Lu H and Gu Z: Pressure and inflammatory stimulation induced increase of cadherin-11 is mediated by PI3K/Akt pathway in synovial fibroblasts from temporomandibular joint. Osteoarthritis Cartilage 21: 1605-1612, 2013.

4. Slade GD, Conrad MS, Diatchenko L, Rashid NU, Zhong S, Smith S, Rhodes J, Medvedev A, Makarov S, Maixner W and Nackley AG: Cytokine biomarkers and chronic pain: Association of genes, transcription, and circulating proteins with temporomandibular disorders and widespread palpation tenderness Pain 152: 2802-2812, 2011.

5. Dai SM, Shan ZZ, Nakamura H, Masuko-Hongo K, Kato T, Nishioka K and Yudoh K: Catabolic stress induces features of chondrocyte senescence through overexpression of caveolin 1: Possible involvement of caveolin 1-induced down-regulation of articular chondrocytes in the pathogenesis of osteoarthritis. Arthritis Rheum 54: 818-831, 2006.

6. Okamoto K, Kiga N, Shinohara Y, Tojyo I and Fujita S: Effect of interleukin-1beta and dehydroepiandrosterone on the expression of lumican and fibromodulin in fibroblast-like synovial cells of the human temporomandibular joint. Eur J Histochem 59: $2440,2015$.

7. Dinarello CA: A clinical perspective of Il-1 $\beta$ as the gatekeeper of inflammation. Eur J Immunol 41: 1203-1217, 2011.
8. Korherr C, Hofmeister R, Wesche H and Falk W: A critical role for interleukin-1 receptor accessory protein in interleukin-1 signaling. Eur J Immunol 27: 262-267, 1997.

9. Yudoh K, Nguyen vT, Nakamura H, Hongo-Masuko K, Kato T and Nishioka K: Potential involvement of oxidative stress in cartilage senescence and development of osteoarthritis: Oxidative stress induces chondrocyte telomere instability and downregulation of chondrocyte function. Arthritis Res Ther 7: R380-R391, 2005.

10. Weng AP, Ferrando AA, Lee W, Morris JP IV, Silverman LB, Sanchez-Irizarry C, Blacklow SC, Look AT and Aster JC: Activating mutations of notch1 in human T cell acute lymphoblastic leukemia. Science 306: 269-271, 2004.

11. Weng AP and Aster JC: Multiple niches for notch in cancer: Context is everything. Curr Opin Genet Dev 14: 48-54, 2004.

12. Selkoe D and Kopan R: Notch and presenilin: Regulated intramembrane proteolysis links development and degeneration. Annu Rev Neurosci 26: 565-597, 2003.

13. Bray SJ: Notch signalling: A simple pathway becomes complex. Nat Rev Mol Cell Biol 7: 678-689, 2006.

14. Eagar TN, Tang Q, Wolfe M, He Y, Pear WS and Bluestone JA: Notch 1 signaling regulates peripheral $\mathrm{T}$ cell activation. Immunity 20: 407-415, 2004.

15. Palaga T, Miele L, Golde TE and Osborne BA: TCR-mediated notch signaling regulates proliferation and IFN-gamma production in peripheral T cells. J Immunol 171: 3019-3024, 2003.

16. Ando K, Kanazawa S, Tetsuka T, Ohta S, Jiang X, Tada T, Kobayashi M, Matsui $\mathrm{N}$ and Okamoto T: Induction of notch signaling by tumor necrosis factor in rheumatoid synovial fibroblasts. Oncogene 22: 7796-7803, 2003.

17. Monsalve E, Pérez MA, Rubio A, Ruiz-Hidalgo MJ, Baladrón V, Garcia-Ramirez JJ, Gómez JC, Laborda J and Diaz-Guerra MJ: Notch-1 up-regulation and signaling following macrophage activation modulates gene expression patterns known to affect antigen-presenting capacity and cytotoxic activity. J Immunol 176: 5362-5373, 2006.

18. Palaga T, Buranaruk C, Rengpipat S, Fauq AH, Golde TE, Kaufmann SH and Osborne BA: Notch signaling is activated by TLR stimulation and regulates macrophage functions. Eur J Immunol 38: 174-183, 2008.

19. Pastrana JL, Sha X, Virtue A, Mai J, Cueto R, Lee IA, Wang H and Yang XF: Regulatory T cells and atherosclerosis. J Clin Exp Cardiolog 2012 (Suppl 12): S2, 2012.

20. Livak KJ and Schmittgen TD: Analysis of relative gene expression data using real-time quantitative PCR and the 2(-Delta Delta C(T)) method. Methods 25: 402-408, 2001.

21. Kou XX, Wu YW, Ding Y, Hao T, Bi RY, Gan YH and Ma X: $17 \beta$-estradiol aggravates temporomandibular joint inflammation through the NF- $\mathrm{NB}$ pathway in ovariectomized rats. Arthritis Rheum 63: 1888-1897, 2011.

22. Aliko A, Ciancaglini R, Alushi A, Tafaj A and Ruci D: Temporomandibular joint involvement in rheumatoid arthritis, systemic lupus erythematosus and systemic sclerosis. Int J Oral Maxillofac Surg 40: 704-709, 2011.

23. Wojdasiewicz P, Poniatowski $Ł A$ and Szukiewicz D: The role of inflammatory and anti-inflammatory cytokines in the pathogenesis of osteoarthritis. Mediators Inflamm 2014: 561459, 2014.

24. Kaneyama K, Segami N, Yoshimura H, Honjo M and Demura N: Increased levels of soluble cytokine receptors in the synovial fluid of temporomandibular joint disorders in relation to joint effusion on magnetic resonance images. J Oral Maxillofac Surg 68: 1088-1093, 2010.

25. Parks WC, Wilson CL and López-Boado YS: Matrix metalloproteinases as modulators of inflammation and innate immunity. Nat Rev Immunol 4: 617-629, 2004.

26. Nascimento GC, Rizzi E, Gerlach RF and Leite-Panissi CR: Expression of MMP-2 and MMP-9 in the rat trigeminal ganglion during the development of temporomandibular joint inflammation. Braz J Med Biol Res 46: 956-967, 2013.

27. Baldwin AS Jr: The NF-kappa B and I kappa B proteins: New discoveries and insights. Annu Rev Immunol 14: 649-683, 1996.

28. Barnes PJ and Karin M: Nuclear factor-kappaB: A pivotal transcription factor in chronic inflammatory diseases. N Engl J Med 336: 1066-1071, 1997.

29. Ogata N, Yamamoto H, Kugiyama K, Yasue H and Miyamoto E: Involvement of protein kinase $\mathrm{C}$ in superoxide anion-induced activation of nuclear factor-kappa B in human endothelial cells. Cardiovasc Res 45: 513-521, 2000.

30. Li Q and Verma IM: Nf-kappaB regulation in the immune system. Nat Rev Immunol 2: 725-734, 2002. 